\title{
Peer to Peer Communication Between the Autonomous Vehicles Using Virtual Private Network
}

\author{
Guruprasad Y K ${ }^{1}$, Dr. Nageswara Guptha $\mathrm{M}^{2}$, Dr. Hema $\mathrm{M} \mathrm{S}^{3}$ \\ \{guruprasad.yk@gmail.com¹,mnguptha@yahoo.com²,ghema_shri@yahoo.co.in ${ }^{3}$ \} \\ Research Scholar, SVCE, Bengaluru1, Associate Professor Senior Grade 2, VIT Bhopal University, \\ Sehore $^{2}$, Associate Professor, Anurag University, Hyderabad ${ }^{3}$
}

\begin{abstract}
Autonomous Vehicles based transportation system is the next generation transportation system in each and every country. All the developing and developed countries are very much interested in developing autonomous vehicle based transportation system to avoid the accidents. As per the statistics taken by the road transportation authorities in USA on an average of $60 \%$ of the accident are happening purely due to the mistakes of drivers. It is almost same in each and every country. Though strict rules and fines imposed still the problems are growing not reducing. The research on autonomous vehicles transportation system has been initiated almost 10 years back still the results are not found fruitful. As the concept of autonomous vehicles is the combination of both Software and Hardware. Though the Hardware Industry is growing day by day with many latest hardware components which includes IC's, Sensors, and IOT Devices etc.., to develop latest hard ware components. The possibility of manufacturing autonomous vehicles is improved a lot further, Countries like China, Singapore, USA already started running the trail autonomous transportation system along with driver assistance, but the biggest challenge faced by this autonomous vehicle transport system is with respect to software. Though the software industry growing day by day with many of the latest technologies like Artificial Intelligence, Machine Learning, Data Science, Big Data etc..,. But the biggest challenge with respect to autonomous vehicles lies in data communication between the vehicles and also identifying the natural disasters. The proposed research mainly concentrated on the cleaning of the collected data from the vehicles, so that the autonomous vehicles Can easily communicate with each other without any delay.
\end{abstract}

Keywords: Artificial Intelligence, Autonomous Vehicles, Big Data, Data Science, IOT, Machine Learning..

\section{Introduction}

Autonomous Vehicles are the next generation vehicles which make life of the people so simple. They not only reduce the burden of the driver it also gives freedom to the people while they travel between the long distances. The Concept of Autonomous Vehicles has been started in the research labs way back almost like 10 Years. The Autonomous vehicles are the combination of both hardware and software. There should be a good understanding between the hardware and software in order to build a very good autonomous vehicle. Consider an example while building autonomous vehicle if the sensor connected to guide the root along with Google Maps. Let's consider if both of them not communicating properly to each other 
obviously the autonomous vehicle either will stop or go further to hit the objects in the road. So that means the coordination between hardware and software is very important in order to build the autonomous vehicle.

The researchers are working parallel to build the best Hard Ware and Software solutions for building the best autonomous vehicles. The major research going on in the field of producing autonomous vehicle based transportation system. The Countries like China, Singapore, Brazil and USA already started doing first level testing on the autonomous vehicle transportation along with driver assistance. Though they are not providing the full fledge autonomous transportation, at least in the areas where there is less traffic, very few signals, Limited Stops along with assistance of the driver who will be handling the vehicle after certain distance by converting it into manual mode. It is clearly understood that building the autonomous vehicles by taking the existing vehicles is not big challenge. Because few companies, are building the autonomous vehicles from the existing vehicles just by adding some additional features and hardware. When the researchers are ready to build autonomous vehicles with existing system and also the software's to communicate with hard ware and software internally built. The major challenges faced by the researchers while building the autonomous vehicle based transportation system is:[3]

- Effective Preparation of Geospatial Data to understand by the Autonomous Vehicles.

- Performing Analytics in the Geospatial Data Received for Decision Making.

- Communicating between the autonomous vehicles for the better transportation.

These are the three major issues which need to be still addressed before making sure that the normal vehicles get replaced with autonomous vehicles for public transport.[4]

\section{Literature Survey}

In this paper, the understanding of what are the possibilities for single vehicle accidents has been studied for future reference.[4]

This paper gave an idea how best the vehicle to vehicle infrastructure can be built for proper communication of data between the vehicles.[5]

In this paper the understanding of Wireless Communication and how fuzzy logic will help in this is understood.[6]

From this paper how the dynamism will be applied between the vehicles for timely delivery of messages will be understood. Which is very much essential for the proper communication between the autonomous vehicles. [7]

This paper gave deep insight on the how intelligent infrastructure will be built amongst the vehicles for proper communication of information and which avoids accidents, [8]

This paper gave a clear idea on how continuous communication can made between the vehicles for proper communication between the vehicles [10]

The main intake from this paper is how inter vehicular communication will be made for proper communication amongst the vehicles for smooth moving on the roads. [14]

This paper gave insight on the how the proper intersection will be made for vehicles to cross each other by communicating to avoid accidents and also unnecessary congestions. [19] 


\section{Illustration}

By considering all above mentioned scenarios and also the research made in the field of autonomous vehicles, it is very clear that there is a need of working on geo spatial data in order to provide the best service in the field of Autonomous Vehicles [14]

And it is very clear that the major problem faced is

- Processing, analyzing and visualizing the geo spatial data.

- Communicating the Data with autonomous vehicles for better transportation.

In this proposed research the major concentrate will be on the how best the geo spatial data can be communicated with the next available vehicles for better transportation. [5]

\section{Geo Spatial Data}

Geo Spatial data is data about the object that lies on the surface of the earth which are either static or dynamic in nature.

In this scenario the vehicles data will be recorded and shared for further use. In day to day world huge geo spatial data is getting generated as so many objects are either dynamic or static. With respect to autonomous transportation system huge geo spatial data is required and which is already captured for further huge. But the biggest challenge is how this data can be understood by the autonomous vehicle for further use

\section{How Geo Spatial Data will help autonomous vehicles?}

The Geo Spatial data will help the autonomous vehicles to identify the shortest path, identify the objects, and communicate the geo spatial data to the next available autonomous vehicle. [8]

In the proposed methodology initially the entire geo spatial data will be collected from the existing resources like woldclim, terafly, usgc etc.., the data which is collected may be structured or un-structured or semi -structured.[24] Once the data has been received then the data will be store in the required format. Along with geo spatial data the help of Google maps will be taken into consideration to provide the required information for the autonomous vehicles.

\section{Proposed Method}

In the proposed methodology, the geospatial information of the vehicles will be recorded in live with respect to all the vehicles. And this data will be further used for peer to peer communication between the autonomous vehicles for better transportation and delay tolerance. In vehicular adhoc networks making vehicle to reach the destination is not important, the main thing here is how best the route has been selected to avoid traffic, natural disasters, unnecessary delays etc.., [6]

In the proposed research main concentration has been given based on the existing technologies, let us consider an example if an autonomous vehicle is passing between the source and destination like A \& B. Most of the autonomous vehicles will follow Google Maps where the longitude and latitude information will be stored in the database. Same will be given access to autonomous vehicles to travel between the source and destination A \& B. Here the 
biggest problem is if an autonomous vehicle is travelling between the source and destination, if there is a huge traffic or some natural disaster happened in that way, it has to be communicated to the all autonomous vehicles coming in the same direction. More than this all autonomous vehicles should fall under one umbrella to communicate with each other for better transportation.[7]

\section{Proposed Research}

In the proposed research one virtual private network has been created with all autonomous vehicles which are travelling in a particular cluster. [2] Here each and every city will be treated as one cluster. If a new autonomous vehicles enters into this cluster it has to be approved by the admin which can be done automatically how a user joins new Wi-Fi network. Once it is approved the vehicle will be tracked till it leaves that particular cluster.[9-12]

\section{Clustering Algorithm:}

- Start

- Create Virtual Private Network

- Create Clusters based on the Longitude and Latitude of the City Limits in VPN.[2]

- Identify the default autonomous vehicles under this cluster.

- If New vehicle enters into this cluster

$\circ \quad$ Check is it Genuine Vehicles based on default parameters.

$\circ$ If it is true

- Allow vehicle to join the cluster

- Start Tracking

Else

- Disconnect the vehicles and send suspected information to nearby officials to take the action.

- Track each and every autonomous vehicle which is moving in the cluster

- Share the required information to the vehicles.

- Repeat the above steps every time till the autonomous vehicles reaches destination.

- Stop

So the above clustering algorithm helps the user to create cluster and identify the autonomous vehicles moving in that particular cluster.[2]

Each and every activity performed by the autonomous vehicle will be tracked from the server side which is connected with in VPN.[13-15] The VPN server has access to communicate with each and every vehicle. Say for example if an vehicles is moving between the Source and Destination, if there is a huge traffic at particular point or some natural disaster happened, if there is slow moving of traffic same information will be shared by the autonomous vehicle to the VPN server and same will be published to the all vehicles in that particular cluster. With this information the autonomous vehicles can opt for different path to reach the destination.[1]

\section{Autonomous Vehicle Communication Algorithm}

- Start

- Joined to Virtual Private Network of particular cluster.

- Travel towards the Destination. 
- If Found Any Disturbance in the path

- Communicate information to VPN server Else

○ Reach the Destination.

- Each and Every Vehicle is tracked in the cluster.

- End

Here the biggest challenge is how fast the autonomous vehicle will communicate to the Server and same will be published to the other vehicles.[21-23]

And how best the autonomous vehicles will understand the message, as they don't have IQ to understand.[1]

In order to avoid this issue the proposed research will follow some mnemonics to communicate effectively between the autonomous vehicles. These default mnemonics will be pre stored in each and every vehicle for comparison to take decision.[16-19]

\section{Mnemonics:}

Mnemonics are nothing but short codes used for the proper understanding and the fast communication.

Along with this mnemonics the longitude and latitude information will be passed to the vehicles. [20]

\begin{tabular}{|l|l|l|l|}
\hline Mnemonic & Description & Longitude & Latitude \\
\hline
\end{tabular}

Table 1. Structure of Mnemonics

Instead of sending the entire data, just send only mnemonic code for easy understanding of the vehicles. Based on mnemonic code the final decision will be taken by the vehicles.[1]

\begin{tabular}{|l|l|l|l|}
\hline Mnemonic & Description & $\begin{array}{l}\text { Longitud } \\
\text { e }\end{array}$ & $\begin{array}{l}\text { Latitud } \\
\text { e }\end{array}$ \\
\hline M1 & Huge Traffic & 64.20001 & 64.3330 \\
\hline M2 & Moderate Traffic & 64.23123 & 65.0232 \\
\hline M3 & Natural Disaster & 62.03231 & 63.0202 \\
\hline
\end{tabular}

Table 2. Sample Mnemonic

By considering all the above algorithms, it will be created as a protocol for better communication and reduce the delay in communicating between the vehicles. The all algorithms and mnemonics will be collected to create protocol for better communication between the vehicles.

\section{Conclusion}

Here in this proposed method the system will be built such a way that the autonomous vehicles will communicate with each other for better transportation. The vehicles will communicate with each other using VPN. The VPN plays vital role while communication information to the different vehicles. 


\section{Future Enhancement}

In future the camera will be fixed for all the autonomous vehicles which would rotate $360^{\circ}$ when there is a mnemonic received from particular vehicles immediately the live scenario will be observed from this camera to understand the scenario.

Word document can be used as a template for papers to be published in EAI Core Proceedings. Follow the text for further instructions on text formating, tables, figures, citations and references.

\section{References}

[1] Pampapathi, B.M., Nageswara Guptha, M. \& Hema, M.S. Data distribution and secure data transmission using IANFIS and MECC in IoT. J Ambient Intell Human Comput (2021). https://doi.org/10.1007/s12652-020-02792-4

[2] Maheshprabhu R, Hema,Srilatha Chepure, Nageswara Guptha, M. Logistics Optimization in Supply Chain Management using Clustering Algorithms Scalable Computing : Practice and Experience 2020,21,107-114

[3] D. Schrank, B. Eisele, and T. Lomax, "TTI's 2012 urban mobility report," Texas A\&M Transportation Institute The Texas A\&M University System, December 2012 (Web Link)

[4] G. Yannis et al., "Traffic safety basic facts 2012: Single Vehicle accidents," European Commission, European Road Safety Observation, Brussels, Belgium, 2013 (Web Link)

[5] J. Miller, "Vehicle-to-Vehicle-to-Infrastructure (V2V2I) intelligent transportation system architecture," in Proceedings of IEEE Intelligent Vehicle Symposium, 2008, pp. 715-720. (Conference)

[6] V. Milanés, J. Pérez, E. Onieva, and C. González, "Controller for urban intersections based on wireless communications and fuzzy logic," IEEE Intelligent Transportation Systems, vol. 11, no. 1, pp. 243-248, Mar. 2010 (Journal)

[7] K. Liu and V. Lee, "Adaptive data dissemination for time-constrained messages in dynamic vehicular networks," Transportation Research Part C: Emerging Technology, vol. 21, no. 1, pp. 214-229, Apr. 2012. (Journal)

[8] S. D. Gupta, Y. P. Fallah, and S. E. Shladover, "Sharing Vehicle and infrastructure intelligence for assisted intersection safety," in Proceedings of 2011 IEEE 22nd International Symposium on Personal, Indoor and Mobile Radio Communications, pp. 767-771. (Conference)

[9] J. A. Misener and S. E. Shladover, "Path investigations in Vehicle-roadside cooperation and safety: A foundation for safety and Vehicle-infrastructure integration research," Proceedings of the IEEE Intelligent Transportation Systems Conference 2006 Pages 9-16. (Conference)

[10] M. Khabazian, M. Mehmet-Ali, and S. Aissa, "Analysis of continuous communication availability in vehicular ad hoc networks," IEEE System Journal, vol. 7, no. 1, pp. 137-150, Mar. 2013. (Journal)

[11] P. Mirchandani and F.-Y. Wang, "RHODES to intelligent transportation systems," IEEE Intelligent System, vol. 20, no. 1, pp. 10-15, Jan/Feb. 2005. (Journal)

[12] "First electric traffic signal installed," History.com. [Online]. Available: (Web Link)

[13] K. M. Dresner and P. Stone, "A multiagent approach to autonomous intersection management," Journal of Artificial Intelligence Research, vol. 31, no. 1, pp. 591-656, Mar. 2008. (Journal)

[14] L. Li and F.-Y. Wang, "Cooperative driving at blind crossings using interVehicle communication," IEEE Transaction Vehicluar. Technology, vol. 55, no. 6, pp. 1712-1724, Nov. 2006. (Journal)

[15] M. R. Hafner, D. Cunningham, L. Caminiti, and D. Del Vecchio, "Cooperative collision avoidance at intersections: Algorithms and experiments," IEEE Transaction Intelligent Transportation Systems, vol. 14, no. 3, pp. 1162-1175, Sep. 2013. (Journal) 
[16] M. R. Hafner and D. Del Vecchio, "Computational tools for the safety control of a class of piecewise continuous systems with imperfect information on a partial order," SIAM Journal on Control and Optimization, vol. 49, no. 6, pp. 2463-2493, Nov. 2011. (Journal)

[17] H. Kowshik, D. Caveney, and P. Kumar, "Provable systemwide safety in intelligent intersections," IEEE Transactions on Vehicular Technology, VOL. 60, NO. 3, MARCH 2011. (Journal)

[18] Jia Wu, A. Abbas-Turki, and A. El Moudni, "Cooperative driving: An ant colony system for autonomous intersection management," Applied Intelligence, vol. 37, no. 2, pp. 207-222, Sep. 2012. (Journal)

[19] J. Lee and B. Park, "Development and evaluation of a cooperative vehicle intersection control algorithm under the connected vehicles environment," IEEE Transaction Intelligent Transportation System, vol. 13, no. 1, pp. 81-90, Mar. 2012. (Journal)

[20] J. Lee, B. B. Park, K. Malakorn, and J. J. So, "Sustainability assessments of cooperative Vehicle intersection control at an urban corridor," Transportation Research Part C: Emerging Technology, Vol. 32, July 2013, Pages 193-206. (Journal)

[21] L. Makarem and D. Gillet, "Model predictive coordination of autonomous Vehicle crossing intersections," Proceedings of the 16th International IEEE Annual Conference on Intelligent Transportation Systems (ITSC 2013), The Hague, The Netherlands, October 6-9, 2013. (Conference)

[22] M. Kamal, J. Imura, T. Hayakawa, A. Ohata, and K. Aihara, "A Vehicle intersection coordination scheme for smooth flows of traffic without using traffic lights," IEEE Transaction Intelligent Transportation Systems, VOL. 16, NO. 3, JUNE 2015. (Journal)

[23] Junqing Wei, John M. Dolan and Bakhtiar Litkouhi. "Autonomous Vehicle Social Behavior for Highway Entrance Ramp Management,” 2013 IEEE Intelligent Vehicles Symposium (IV) June 23-26, 2013, Gold Coast, Australia (Journal)

[24] Junko Yoshida. 2016. Autopilot: Flying vs. Driving. Can lessons learned from aviation apply to cars? EE Times. Retrieved December 10, 2016 from (Web Link) 